\title{
Multi-objective Optimisation for Small Meander Wire Dipole Antennas in a Fixed Area Using Ant Colony System.
}

\author{
Amir Galehdar, David V. Thiel, Andrew Lewis", Marcus Randall* \\ Centre for Wireless Monitoring and Applications, Griffith University, Nathan Qld \\ 4111 Australia \\ \# Institute for Integrated and Intelligent Systems, Griffith University, Nathan Q1d \\ 4111 Australia \\ *School of Information Technology, Bond University, Qld 4229, Australia
}

\begin{abstract}
Fixed area meander line antennas designed on a 5 x 5 rectangular grid were optimised for efficiency and resonant frequency. The Pareto front was developed using ant colony optimisation and the numerical electromagnetic code (NEC). The lowest frequency antenna fits an area of $0.11 \lambda_{0} \times 0.05 \lambda_{0}$ with an efficiency of $88 \%$.
\end{abstract}

Keywords: meander line antenna, ant colony optimisation, RFID, backbite operator, printed antennas, Pareto front.

\section{Introduction:}

Optimised antenna design is now essential for mass market applications both in terms of the space occupied, the materials used and the antenna efficiency. Mass market applications include RFID (radio frequency identification devices), WSNs (wireless sensor networks), remote controlled toys, and keyless entry for automobiles, garage doors, and building access doors. Many appliance manufacturers choose to use frequencies in the UHF band because the radio link can operate satisfactorily without line-of-site between the transmitter and receiver. The UHF spectrum spans the frequency range from $300 \mathrm{MHz}$ to $3 \mathrm{GHz}$ with a free space wavelength range from 1 $\mathrm{m}$ down to $100 \mathrm{~mm}$. Traditional antennas require structures approximately $0.5 \lambda_{0}$ (where $\lambda_{0}$ is the free space wavelength) which is far too large for most of the applications.

Electrically small antennas are defined as antennas that "can be contained in a sphere whose diameter is small compared to a wavelength at the frequency of operation" [1]. More specifically the definition has been refined in terms of a radiansphere [2] where the radius of the sphere is $\lambda_{0} / 2 \pi$. The size of a small antenna is often quoted in terms of $k r$ where $k=2 \pi / \lambda_{0}$ is wave number and $r$ is the radius of a sphere enclosing the antenna. The Wheeler definition [2] is $k r \leq 1$.

Small antennas have clearly defined performance limits to their efficiency [3]. The input impedance of this class of antennas is characterised by a relatively small real part and a much larger reactive part. The antennas therefore provide a very poor match to typical transmission lines (e.g $50 \Omega$ coaxial line). An alternative method of defining the minimum possible bandwidth $B W$ of an antenna has been adopted in terms of the $Q$ factor of the antenna [4]; that is $B W \cong 1 / Q$. When the efficiency $\eta$ is less than $100 \%$, this relation can be written as: 


$$
B W=\frac{1}{\eta Q}
$$

Choo et al [5] defined a figure of merit $\psi$, using the equation:

$$
\psi=\frac{\eta B W}{\left(\frac{2}{Q}\right)}
$$

and the fundamental limit for $\psi$ is always unity. This expression requires an alternative definition of $B W$ (see [6]).

Choo et al [5] removed this restriction and showed that an impedance in the range of $1 \Omega$ to $300 \Omega$ did not change the results significantly. In this paper the same principle was used and the band width of each antenna is determined by the $-3 \mathrm{~dB}$ variation in S11 as given by:

$$
S 11=20 \log \left|\frac{R_{\text {in }}-Z_{\text {in }}\left(f_{0}\right)}{R_{\text {in }}+Z_{\text {in }}\left(f_{0}\right)}\right|
$$

where the $R_{i n}$ is the input resistance of the antenna at resonance. This can differ between antennas. The $-3 \mathrm{~dB}$ point occurs when $2 R_{i n}=+X_{i n}$ and $Z_{i n}=R_{i n}+j X_{i n .}$.

The task in antenna optimisation is to design structures which approach this limit as closely as possible. Choo et al [5] used a genetic algorithm optimisation technique to design electrically small wire antennas with seven straight line wire segments which fit into a hemispherical volume of radius with $0.29<k r<0.63$ located on an infinite ground plane. With a target frequency of $400 \mathrm{MHz}$, antennas with efficiencies of up to $97 \%$ were constructed to match a $50 \mathrm{ohm}$ transmission line. Best [7] conducted a comprehensive study of electrical small self-resonant wire antennas in a finite volume. Erentok, and Ziolkowski [8] used optimisation prroccedures fpor electroically small antennas on an electronic band-gap ground plane. This style of antenna has some practical disadvantages including:

- The structure is three dimensional and has finite thickness. This increases manufacturing complexity.

- The antennas were modelled on an infinite ground plane. In practice the ground plane must be finite. The antenna is most responsive above the ground plane and so communications below the ground plane are impaired.

There are significant manufacturing advantages in using planar antennas; that is, antennas which are large in two dimensions but very small in the third dimension. The use of a flexible substrate such as plastic film, allows the antenna to conform to the mounting surface. Such antennas have good radiation characteristics both above and below the plane providing they are not placed against a conducting material. Meander line antennas are one form of dipole thin wire antennas in which the wire in one arm is a mirror image of the wire in the other arm. Usually these antennas have straight wire segments laid out on a rectangular grid [9]. Note that meander line antennas do not have a ground plane. 
Using a small number of grid points Galehdar et al [10] exhaustively solved the complete population of antennas using a $5 \times 5$ square grid for one half of the dipole. The wire was required to pass through each grid point once only. More recently the ant colony optimisation routine was used to explore a larger grid pattern and then refined using the backbite operator [11, 12]. These designs were constrained to include every point in the square grid.

In this paper, this requirement was relaxed to allow the antenna wire to terminate before all grid points were included. The two objectives were to maximise efficiency and minimise resonant frequency. In addition, all optimised data was retained to form a two dimensional Pareto front in both antenna efficiency and resonant frequency. This paper outlines the computational approach used and the design improvements achieved by allowing this additional design freedom. The efficiency of the optimisation procedure should allow structures with an increased grid size to be solved.

\section{Antenna Design}

The ant colony algorithm was used to construct a meander wire antenna element by connecting between a set of grid points on a square grid. The grid point separation was $6 \mathrm{~mm}$ so the total area of the dipole antenna was $54 \mathrm{~mm} \times 24 \mathrm{~mm}$ for the 50 grid points (see Fig 1). The antenna feed point was placed at one half of the grid spacing at one of three possible points, as shown. The left arm of the dipole was always a mirror image of the right. The wire diameter was $1 \mathrm{~mm}$. The conductivity of copper $(5.7 \mathrm{x}$ $10^{-7} \mathrm{~S} / \mathrm{m}$ ) was used in the NEC model [14]. Modelling the printed antenna as a thin wire in free space is a common technique when the support material (i.e. the plastic film) is very thin.

The ant colony algorithm used to construct each antenna was started at the point closest to the feed point and passed through a grid point once only. The wire was extended until no additional segment was possible. Thus the shortest achievable antenna half length was $33 \mathrm{~mm}$ and the longest possible half length was $147 \mathrm{~mm}$. Segments constructed by the ant colony algorithm were further developed using a local refinement method [12].

The resonant frequency $f_{0}$ was determined by calculating the antenna impedance at a number of sampled points between $300 \mathrm{MHz}$ and $2000 \mathrm{GHz}$. A binary search method was used to find the frequency where the magnitude of the reactive part of the impedance was minimum, chosen as the resonant frequency. The resolution of the resonant frequency calculations was $\pm 5 \mathrm{MHz}$. 


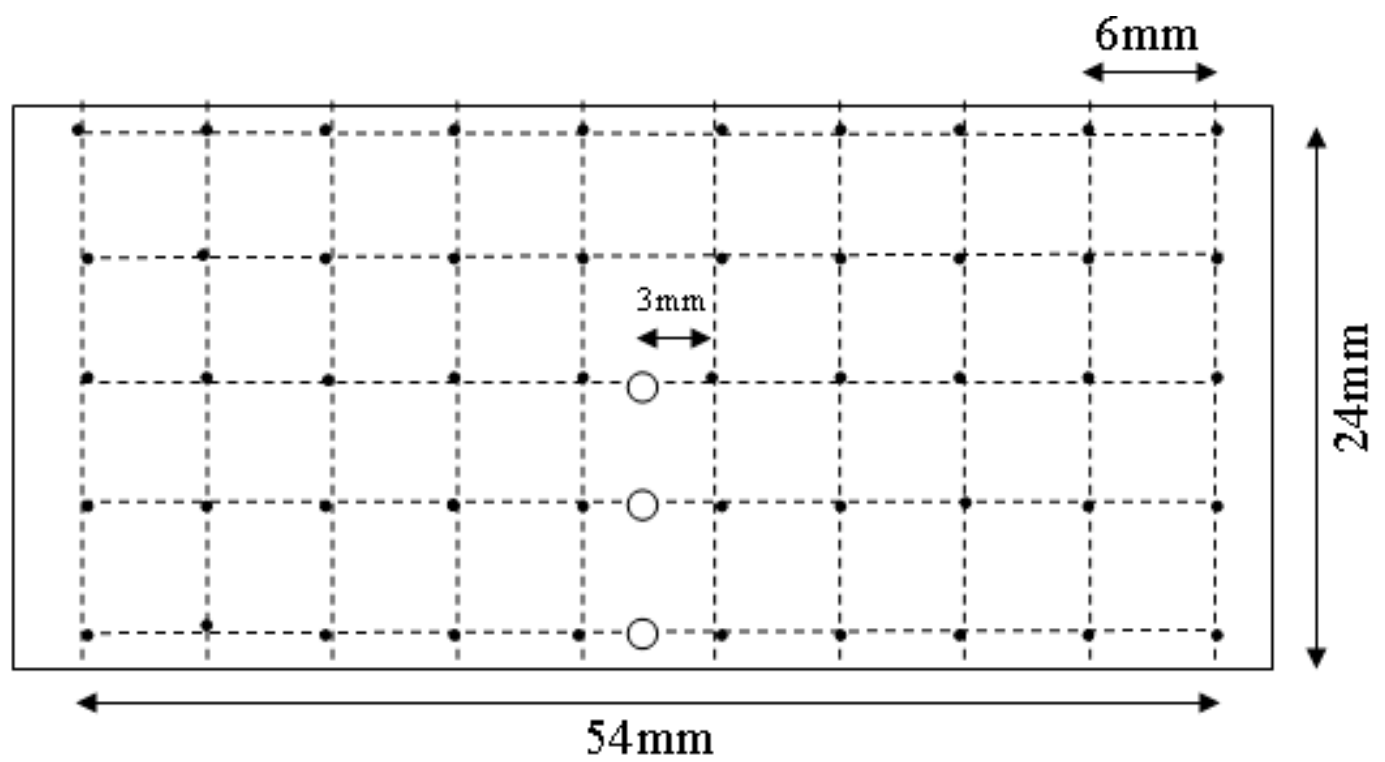

Fig 1. Meander line dipole template. The square grid points were spaced $1 \mathrm{~mm}$ apart. The three possible feed points are shown as open circles along the line of symmetry.

\section{Optimisation Protocol}

The antenna design problem addressed required the development of a continuous meander line path. Few optimization techniques are suitable for random or guided path development. The ant colony optimisation technique applied to the design of meander line antennas was introduced by Randall et al [11]. ACO is modelled on the foraging behavior of Argentine ants. The seminal work by Dorigo [14] showed that this behavior could be used to solve discrete optimisation problems. The method is particularly suited to path planning problems [15] but its use to date for antenna design has been limited.

For the construction of RFID antenna segments, ants placed on the Cartesian grid move from one node to another, subject to a number of heuristic rules. Ants deposit pheromone on the links they traverse to communicate their utility to the colony. Ants have a uniform probability of deviating from paths biased by pheromone concentrations only the length of the segment by use of a pseudo-proportional selection rule, reducing the chance of the optimisation process becoming trapped in local optima.

In addition to being guided by pheromone trails, ants also use a look ahead function to maximise the number of alternative paths away from the node to which they are moving, and may bias paths toward "straight-line" segments to reduce the incidence of premature termination, trapped in small inward-spiraling paths. These rules make use of problem-specific heuristic information to improve algorithm performance.

Local search is widely applied to ACO, generally making significant improvements to solution quality $[15,16]$. As the objective function is essentially a black box, local search is difficult. However, the algorithm developed for this problem uses a form of 
the backbite operator to refine the end segments of near-optimal solutions, exploring a tree of slightly modified segment designs [12].

For the antenna design problem being considered both the antenna efficiency, $\eta$, and the resonant frequency, $f_{0}$, were optimised. When using ACO to optimise a single design objective, it is simple to determine which ant has achieved the best solution; it is the ant whose solution has the best value for the objective. However, when more than one objective is involved, the question of what is the "best" solution can be difficult to answer, particularly if objectives conflict. In this antenna problem it was found that the resonant frequency and efficiency conflict. What is ideally required in this case is a method that delivers information on the trade-off between these two objectives.

To determine whether one solution is more attractive than another, a domination relation may be used. For the solution vectors $\vec{x}_{1}$ and $\vec{x}_{2}$, when the following conditions are met:

○ $\vec{x}_{1}$ is at least as good as $\vec{x}_{2}$ for all the objectives, and

○ $\vec{x}_{1}$ is strictly better than $\vec{x}_{2}$ for at least one objective

then $\vec{x}_{1}$ is said to "dominate" $\vec{x}_{2}$. In the case where $\vec{x}_{1}$ and $\vec{x}_{2}$ dominate other solution vectors but not each other they are deemed mutually optimal solutions and referred to as Pareto-optimal. The set of Pareto-optimal solutions reflects the tradeoff surfaces between the different objectives. This set of Pareto-optimal solutions is referred to as the Pareto-front.

This approach to optimisation of multiple objectives delivers not just a single solution but a set of (Pareto-optimal) solutions. Design engineers must still make some decision as to which particular solution is most fit for the requirements of a specific application.

\section{Results}

Fig 2 shows the $\eta f_{0}$ Pareto front for the square grid meander line antenna shown in Fig 1. The resonant frequency range was $602 \mathrm{MHz}<f_{0}<1610 \mathrm{MHz}$ and efficiency range was $88 \%<\eta<99.3 \%$. The highest density population was found in the frequency range between $740 \mathrm{MHz}$ and $990 \mathrm{MHz}$ which equates to an effective antenna size of between $0.57<k r<0.72$. The Pareto plot is replotted using efficiency $\eta$ and antenna size $k r$ in Fig 3.

The data was converted to the figure of merit and replotted as a function of antenna size (see Fig 4). The figure of merit was found to lie in the range $0.05<\psi<0.22$. Curiously the relationships appears almost linear $(\psi=0.3 k r+0.08$, with a correlation coefficient $s^{2}=0.98$ ). While no reason is apparent why this relationship should be linear, the relationship does allow antenna designers to evaluate the strength of a planar meander line antenna structure for a fixed area. It is important to note that $\psi$ contains band width information which was not used in the optimisation process, and so it is possible for some antennas to lie above the straight line given in Fig. 4. 
Using multi-parameter optimisation, a three dimensional dual seven segment monopole achieved a figure of merit of almost $\psi=0.9$ for $k r=0.62$ [5]. This design however, required a ground plane and was optimised throughout a hemisphere of this radius. Intuitively, a planar structure can not achieve such high values.

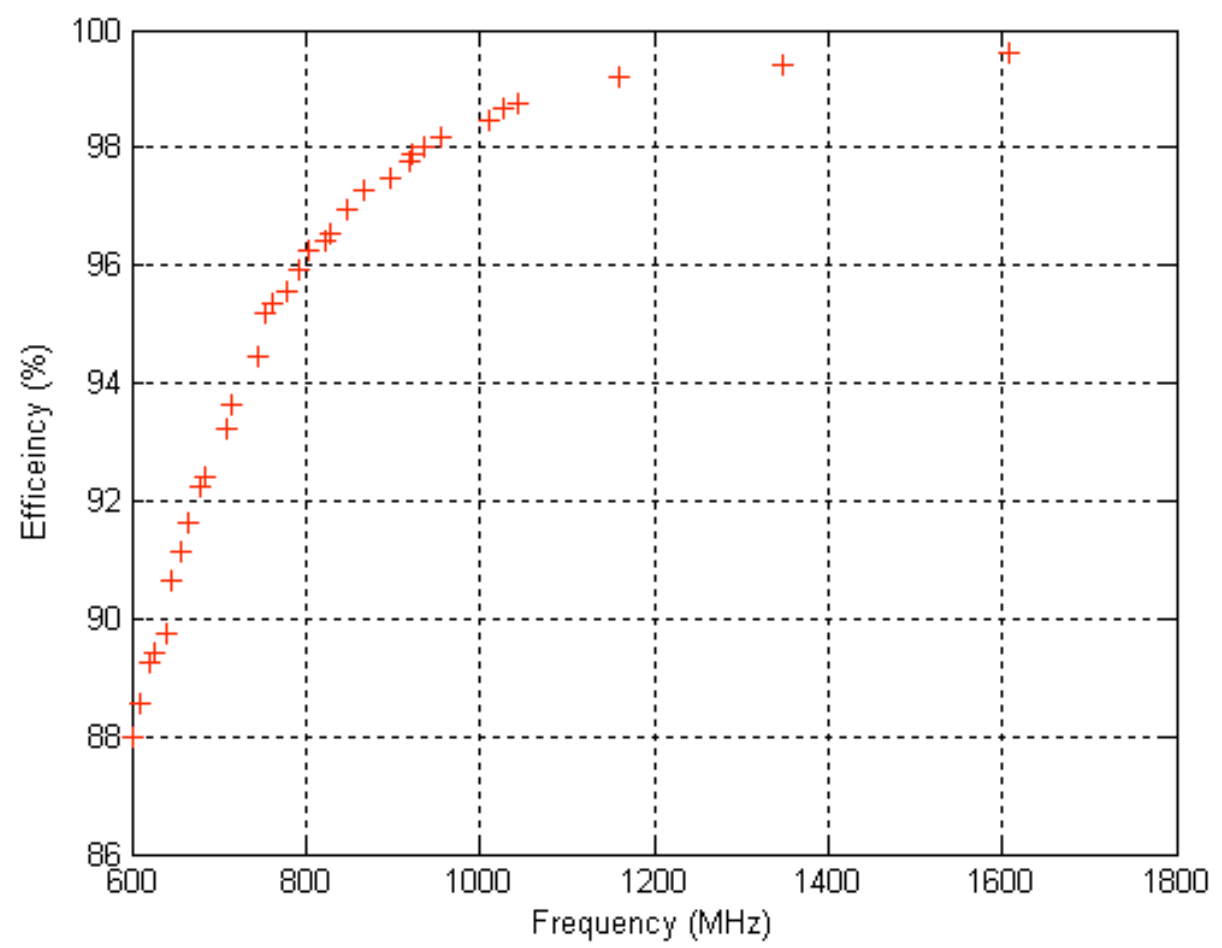

Fig 2: The $\eta f_{0}$ Pareto front

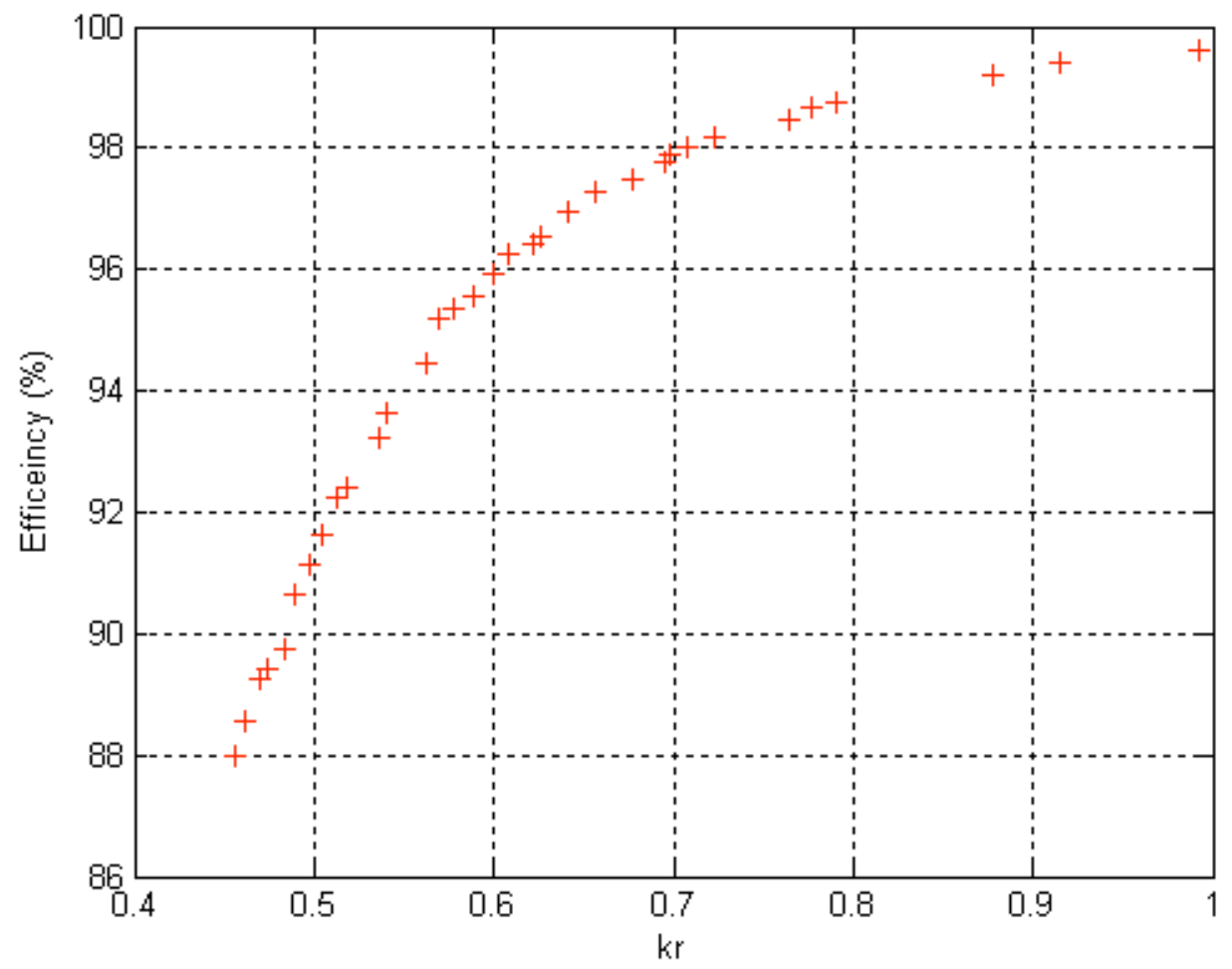

Fig 3: The Pareto front from Fig 2, replotted using $\eta$ but with $f_{0}$ replaced by the effective size of the antenna $k r$. 


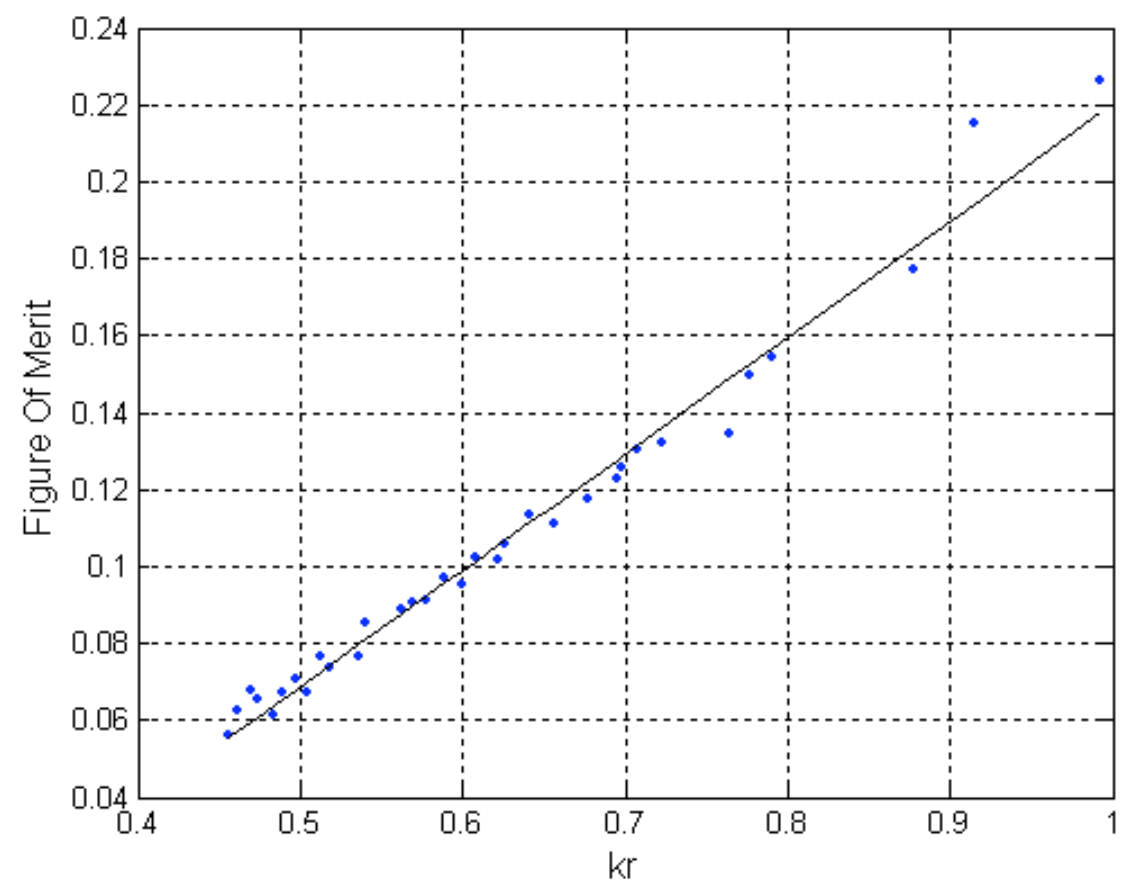

Fig 4: Pareto front plotted as a figure of merit $\psi$ defined by equation (3). A straight line of best fit has been included.

The antennas with the highest resonant frequency are part of the family shown in Fig $5 \mathrm{a}$, and those antennas with the lowest resonant frequency have the shortest allowable lengths (Fig 5b). The lowest resonant frequency is achieved when adjacent high current segments have current in the same direction $[10,12]$.
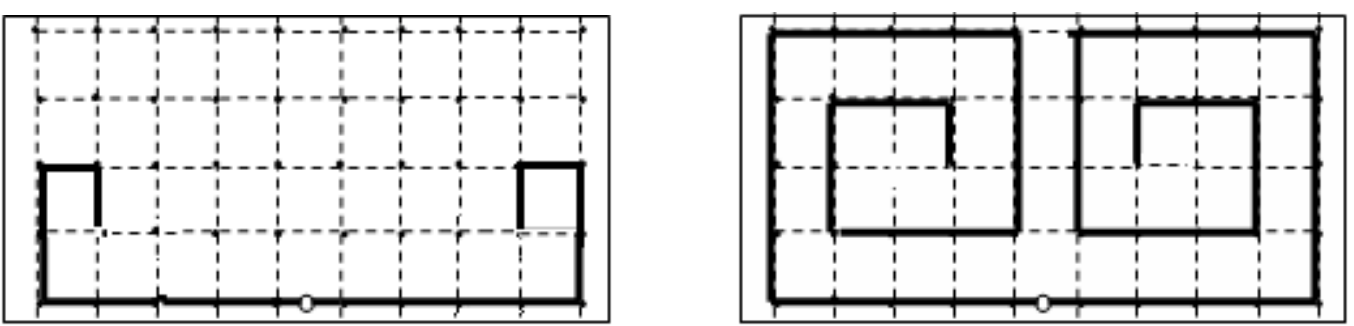

Fig. 5 (a) Highest resonant frequency structure $f_{0}=1607 \mathrm{MHz}$, (b) lowest resonant frequency structure $f_{0}=602 \mathrm{MHz}$.

\section{Discussion and Further Work}

During the course of the optimisation, over 13,000 evaluations of dipole structures were carried out by NEC. A population of 10 ants were run 10 times with different, random, initialisation seeds. Each run was permitted 1000 iterations. Local refinement using the backbite operator was permitted to search the tree of structure permutations generated to a depth of three. This obviously involves many more antenna structures than the number submitted to NEC for evaluation. However, the ants (and subsequent 
local refinement) often produced duplicate structures that had already been evaluated. To minimise wasted computation time, a cache of previously-computed performance results was maintained and the program checked candidate solutions against this before sending them for evaluation.

The computations were performed on a collection of some 14 computers: a mix of Intel P4 and Athlon X64 dual-core-based machines. The computers used were not dedicated to this problem but were given tasks based on their ability to process them. Each structure evaluation required between 0.5 and 0.8 seconds computation time, depending on the processing capability. The whole experiment required a little over 16 minutes to complete.

Further work is being directed to optimizing the solution using a fixed area with an increasing number of grid points and including the band width using the figure of merit as used in [5]. Additional challenges follow the optimization of three dimensional rectangular grid meander line antennas [19] and wire antennas with varying segment wire radius [20].

\section{References:}

[1] IEEE, IEEE Standard Definitions of Terms for Antennas. IEEE Std 145(1993).

[2] H.A. Wheeler, Small antennas. IEEE Trans. Antennas and Propagation 23 (1975), 462-469.

[3] J.L. Chu, Physical limitations of omni-directional antennas. J. Appl. Phys. 19 (1948), 1163-1175.

[4] J.S. McLean, A re-examination of the fundamental limits of the radiation Q of electrically small antennas. IEEE Trans. Antennas and Propagation, 44 (1996), 672-676.

[5] H.S. Choo, R.L. Rogers, and H Ling, Design of electrically small wire antennas using a pareto genetic algorithm, IEEE Trans. Antennas and Propagation, 53 (2005) 1038-1046.

[6] E. E. Altshuler, Electrically small self-resonant wire antennas optimized using a genetic algorithm, IEEE Trans. Antennas Propagation 50 (2002) 297-300.

[7] S.R. Best, A discussion on the properties of electrically small self-resonant wire antennas, IEEE Antennas and Propagation Magazine, 46 (2004), 9-22.

[8] A. Erentok, and R.W. Ziolkowski, A hybrid optimisation method to analyse meta-material-based electrically small antennas, IEEE Trans. Antennas Propagation 55 (2007) 731-741.

[9] G. Marrocco, Gain-optimised self-resonant meander line antennas for RFID applications. IEEE Antennas and Wireless Propagation Letters, 2 (2003) 302305.

[10] A. Galehdar, D.V. Thiel., S.G. O'Keefe, and S. Kingsley. Efficiency variations in electrically small, meander line RFID antennas." IEEE Antennas and Propagation International Symposium: (2007), 2273 - 2276.

[11] M. Randall, A. Lewis, A. Galehdar and D.V. Thiel, Using ant colony optimisation to improve the efficiency of small meander line RFID antennas. Proc. Third IEEE Int. Conf. e-Science and Grid Computing, (2007) 345-351. 
[12] G. Weis, A. Lewis, M. Randall, A. Galehdar, and D.V. Thiel, Local search for ant colony system to improve the efficiency of small meander line RFID antennas. WCCI Proc. World Congress Computational Intelligence CEC (2008).

[13] NEC-Win Plus, User's Manual, Nittany Scientific Inc, (2003).

[14] M. Dorigo, Optimization, learning and natural algorithms, $\mathrm{PhD}$ Thesis, Politecnico di Milano, (1992).

[15] M. Dorigo and T. Stützle, Ant colony optimization, MIT Press (2004).

[16] M. Dorigo and L. Gambardella, Ant colony system: A cooperative learning approach to the travelling salesman problem, IEEE Trans. Evolutionary Computation, 1 (1997) 53-66.

[17] T. Stützle, The max-min ant system and local search for combinatorial optimisation problems. In S. Voss, S. Martello, I. Osman, and C. Roucairol, ed., Meta-heuristics: advances and trends in local search paradigms for optimisation, IEEE Press (1999), 309-314.

[18] A. Galehdar, D.V. Thiel and S.G. O'Keefe, Antenna efficiency calculations for electrically small RFID antennas. IEEE Antennas and Wireless Propagation Letters, 6 (2007) 156-159.

[19] A. Galehdar, D.V. Thiel and S. O'Keefe, Design methods for 3D RFID antennas located on a conducting ground plane, IEEE Trans. Antennas and Propagation, (2009) accepted.

[20] A. Galehdar, D.V. Thiel, and S.G. O'Keefe, Tapered wire antenna design for maximum efficiency and minimal environmental impact, ISAPE (2008) Kun Ming, China. 